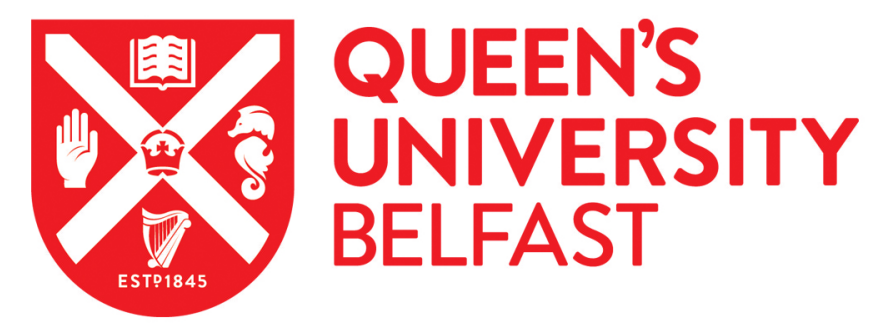

\title{
Technique for Pre-Compliance Testing of Phasor Measurement Units
}

\author{
Brogan, P. V., Laverty, D. M., Zhao, X., Hastings, J., Morrow, D. J., \& Vanfretti, L. (2018). Technique for Pre- \\ Compliance Testing of Phasor Measurement Units. International Journal of Electrical Power and Energy \\ Systems, 99, 323-330. https://doi.org/10.1016/j.ijepes.2018.01.031
}

\section{Published in:}

International Journal of Electrical Power and Energy Systems

\section{Document Version:}

Peer reviewed version

\section{Queen's University Belfast - Research Portal:}

Link to publication record in Queen's University Belfast Research Portal

\section{Publisher rights}

Copyright 2018 Elsevier.

This manuscript is distributed under a Creative Commons Attribution-NonCommercial-NoDerivs License

(https://creativecommons.org/licenses/by-nc-nd/4.0/), which permits distribution and reproduction for non-commercial purposes, provided the author and source are cited.

\section{General rights}

Copyright for the publications made accessible via the Queen's University Belfast Research Portal is retained by the author(s) and / or other copyright owners and it is a condition of accessing these publications that users recognise and abide by the legal requirements associated with these rights.

Take down policy

The Research Portal is Queen's institutional repository that provides access to Queen's research output. Every effort has been made to ensure that content in the Research Portal does not infringe any person's rights, or applicable UK laws. If you discover content in the Research Portal that you believe breaches copyright or violates any law, please contact openaccess@qub.ac.uk. 


\title{
Technique for Pre-Compliance Testing of Phasor Measurement Units
}

\author{
Paul V. Brogan ${ }^{1}$, David. M. Laverty ${ }^{1 *}$, Xiaodong Zhao ${ }^{1}$, John Hastings ${ }^{1}$, D. John Morrow ${ }^{1}$, \\ and Luigi Vanfretti ${ }^{2}$ \\ ${ }^{1}$ School of EEECS, Queen's University Belfast, Belfast, United Kingdom \\ ${ }^{2}$ Rensselaer Polytechnic Institute, Troy, New York, USA \\ *david.laverty@qub.ac.uk (corresponding author)
}

\begin{abstract}
This paper introduces a technique for 'pre-compliance' testing of Phasor Measurement Units (PMUs) against the dynamic requirements of the IEEE C37.118.1-2014 standard, which include dynamic and steady-state test scenarios. . The tests described are a necessary, but not complete, requirement for passing the IEEE standard and quickly highlight shortcomings in PMU operation during dynamic conditions. The pre-compliance test presented in this paper only requires typical relay test equipment, with little requirement for significant temporal accuracy when initiating waveform test files. The compliance test is intended to allow PMU owners to assess a device's performance before considering its use in monitoring dynamic performance. Failure of these tests can indicate the need to recalibrate or replace the PMU or find another vendor. The described method is applied to the voltage inputs of a typical commercial PMU and the results presented. The process for the creation of test waveforms is described, along with the data analysis technique used. The test waveforms and analysis source code are made available under open source licenses.
\end{abstract}

\section{Keywords: Phasor Measurement Unit; Synchrophasor; Compliance Test; Dynamic Performance}

\section{Introduction}

Phasor Measurement Units (PMU) provide very useful measurements for the analysis of electrical power systems. Over the last decade, PMU technology has seen considerable deployment across transmission systems. In more recent years a broad spectrum of applications where PMUs can be exploited in the distribution network, including monitoring, protection and control, have been proposed [1], [2], [3]. In these situations, the value of a PMU greatly exceeds its cost and failure of a PMU can result in missed opportunities and lost man-hours.

Many companies and institutions purchase PMUs with a degree of trust that the PMU they purchase meets particular standards. Research organisations may also operate PMUs outside their intended purposes and wish to know how well the device performs. Usually expensive equipment, with microsecond precision, is required to accurately test PMUs. In this paper, a method of achieving similar results on relatively common relay test equipment is presented.

By definition, phasors are only truly accurate when describing time invariant signals [4]. Therefore, there is a need to ensure uniformity in phasor estimation between PMUs for use with critical infrastructure. The IEEE has addressed this issue through the release of the C37.118.1a2014 [6] standard, and its 2011 predecessor [5]. The C37.118.1 standard specifies how the error of PMU measurements is calculated and states maximum permissible errors under described steady-state and dynamic test conditions. The dynamic tests specify changes in bulk properties of the sinusoidal wave, such as magnitude, frequency and phase, and do not consider harmonic behaviour.

Although the IEEE dynamic standards have been in existence for over six years, at the time of writing, many
PMUs in the marketplace commonly cite compliance against the prior version of the standard, C37.118-2005 - this edition does not mandate dynamic performance. Some devices may have been designed prior to the 2011 edition while other may struggle to meet the exacting standard; consequently their performance under dynamic scenarios is not specified by the manufacturer. Many utility companies will own and operate PMUs manufactured prior to the 2011 standard and may wish to test their performance. Other PMU operators question the consistency of phasor estimation between PMUs of differing designs, as in [6], [7], [8].

The present authors sought out and developed a technique for pre-compliance testing of PMUs against the requirements of the 2014 edition of the IEEE C37.118.1 standard. The requirements were:

- Can be applied with standard test equipment

- Widely available waveform development environment

- Assess the performance of a PMU under dynamic tests

- Be a necessary requirement for passing C37.118.1 tests

This paper describes how test waveforms have been generated to represent the dynamic test scenarios described in the C37.118.1a-2014 standard. These three-phase waveforms are applied to a commercially available PMU and the estimated synchrophasors are recorded. Following this, we describe how the PMU's estimated phasors can be compared against the theoretical phasors [5] without need for GPS synchronization of the test equipment. The performance of the physical PMU is discussed and compared against the synchrophasor that produced the waveform sample data. Errors in synchrophasor estimation are compared against the $\mathrm{C} 37.118 .1 \mathrm{a}-2014$ requirements. As a sanity check the phasor estimation algorithm described in [9] was applied to the raw point on wave data files and it was found to be as accurate as described in that publication. 
The technique presented aims to give PMU owners a cost effective method of determining the dynamic characteristics of their PMUs. PMU owners can then make comparisons between vendors, identify degradation in PMUs and determine if costly compliance testing or recalibration is required. In this way, PMUs suitable for protection, control and analysis applications can be identified.

\section{Compliance Test Specifications}

Test specifications for PMU devices are described in IEEE Std C37.118-2011 [5], with amendments in the 2014 update [6]. The standard describes permissible error limits for PMUs under both nominal and dynamic conditions. Phasor estimation algorithms usually expect cyclical, time invariant waveforms. Distortions in the waveform, due to system transients and other operation behavior, cause the input to the phasor estimation algorithm to be time variant, thus the estimation is of reduced accuracy.

Phadke describes in [4], [6], [10] the problem of estimating phasors under dynamic conditions and reaches the conclusion that either a set of input signals should be described for which the performance of PMUs is defined, as is the approach taken in IEEE Std. C37.118.1-2011, or alternatively the phase estimation algorithms should be uniformly specified.

The IEEE standard defines two classes of PMU, Mclass and P-class. P-class PMUs are optimized for accuracy in a dynamic environment, such as the bandwidth and step tests in Subclause 5.5.6 and 5.5.8; whilst M-class PMUs are expected to remain accurate over a wider range of frequencies (Subclause 5.5.6 and 5.5.7). Maximum permissible errors are mandated for each class of PMU under the following categories:

1) Steady-state

2) Measurement bandwidth

3) Ramp in frequency

4) Step change in phase / magnitude

The C37.118.1 standard describes how these conditions should be applied and assessed.

\subsection{Total Vector Error}

The accuracy of an estimated phasor is expressed as the Total Vector Error (TVE), in percent. TVE is a function of both magnitude error and phase angle error. The TVE is derived from the vector separating the theoretically applied phasor and the estimated phasor, see Fig. 1. The resultant vector magnitude is normalized by dividing it by the theoretical vector magnitude, giving the TVE.

A convenient method for calculating TVE, from phasors in polar format, is presented in (1); this utilises the small angle approximation in radians and is shown graphically in Fig. 1. For small phase error ( $d \phi$ in radians) and with estimated magnitude $(\hat{X})$ approximately equal to theoretical magnitude $(X)$; the equation for TVE, from [5], can be rewritten as shown in (1). The approximation has a maximum error of $-6.75 \times 10^{-4} \%$ when TVE $=3 \%$ due to a $\mathrm{d} \phi=0.03 \mathrm{rad}$; below these values, the error is less.

Under steady-state conditions, the maximum permissible TVE is $1 \%$. This means that if the amplitude error is $1 \%$, phase error must be $0^{\circ}$. If amplitude error is $0 \%$, the maximum permissible phase error is $\pm 0.573^{\circ}(0.01 \mathrm{rad})$. The standard gives definitions of the permissible error limits under each of the test conditions.

$$
\operatorname{TVE}(\%)=[100 / X] \times \sqrt{(X-\hat{X})^{2}+(\hat{X} \times d \phi)^{2}}
$$

\subsection{Measurement Bandwidth}

Measurement bandwidth is assessed by applying sinusoidal amplitude and phase modulation to a set of balanced three-phase voltage and current waveforms. This is expressed mathematically in [5] as shown in Eq (2), the revised application of $\mathrm{Eq} \mathrm{(2)} \mathrm{in} \mathrm{the} \mathrm{test} \mathrm{environment} \mathrm{is}$ described in [6].

$$
X_{1}=X_{m}\left[1+k_{x} \cos (\omega t)\right] \times \cos \left[w_{0} t+k_{a} \cos (\omega t-\pi)\right]
$$

where, $X_{I}$ is the positive sequence component $X_{m}$ is the amplitude of the input signal $\omega_{0}$ is the nominal frequency of the power system $\omega$ is the modulation frequency in radians/s $k_{x}$ is the amplitude modulation factor $k_{a}$ is the phase angle modulation factor

The maximum TVE over the range of measurement bandwidth tests (Sub 5.5.6) must not exceed 3\%. P-class PMUs are to be assessed in the range from $0.1 \mathrm{~Hz}$ to the lesser of $2 \mathrm{~Hz}$ to $\mathrm{Fs} / 10(5 \mathrm{~Hz}$, where Fs is PMU reporting rate, in this case 50 frames per second); M-class PMUs are assessed to the lesser of $5 \mathrm{~Hz}$ to $\mathrm{Fs} / 5(10 \mathrm{~Hz})$. The accuracy of frequency and Rate-of-Change-of-Frequency (ROCOF) estimation are also stipulated for this test [6].

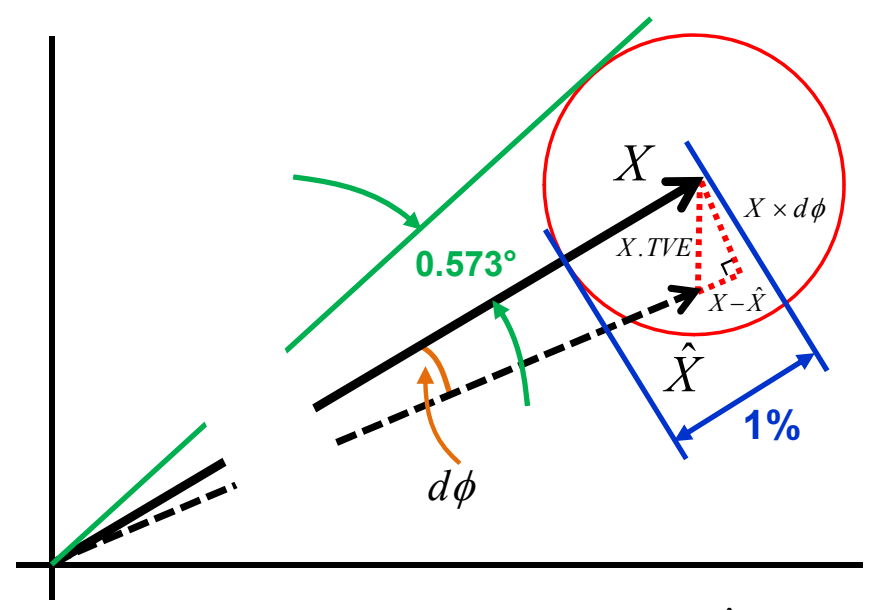

Fig. 1. Permissible region for estimated phasor, $\hat{X}$, shown as a circle around the theoretical phasor, X. Maximum magnitude error is $1 \%$, maximum phase error is $0.573^{\circ}$ (0.01 rad). Pythagoras' Theorem can be used to calculate TVE.

\subsection{Ramp in Frequency}

PMUs are subjected to a linear ramp in system frequency, applied as balanced three-phase input signals. The positive sequence signal corresponding to this test is described mathematically in [5] as shown in Eq (3): 


$$
X_{1}=X_{m} \cos \left[\omega_{0} t+\pi R_{f} t^{2}\right]
$$

where, $X_{I}$ is the positive sequence component

$X_{m}$ is the amplitude of the input signal

$\omega_{0}$ is the nominal frequency of the power system

$R_{f}$ is the frequency ramp rate in $\mathrm{Hz} / \mathrm{s}(\mathrm{df} / \mathrm{dt})$

Tests are started with $100 \%$ rated signal amplitude and at nominal frequency. Ramps are applied at rates of $\pm 1.0 \mathrm{~Hz} / \mathrm{s}$ (positive and negative). For Synchrophasor estimation, in order to be compliant, a P-class PMU must maintain 1\% TVE over a range of $\pm 2 \mathrm{~Hz}$ from nominal frequency and an M-class PMU must maintain 1\% TVE in the range $\pm 5 \mathrm{~Hz}$ or $\pm(\mathrm{Fs} / 5)$, whichever is the lesser.

For a PMU with a reporting rate of 50 frames per second (Fs) the following requirements apply. P-class units must track frequency during the ramp to within $0.4 \mathrm{~Hz}$. Mclass units must track frequency during the ramp to within 0.01

Errors that occur during the measurement exclusion interval [6] are ignored; these exclusions centre on the inflection points when the frequency ramp inverts.

\subsection{Step change in phase / magnitude}

Step changes in phase angle and magnitude are applied in order to determine response time, delay time and overshoot in the measurement. The tests are applied as a transition between two steady-state conditions. This is expressed mathematically in [5] as shown in Eq (4):

$$
X_{1}=X_{m}\left[1+k_{x} f_{1}(t)\right] \times \cos \left[\omega_{0} t+k_{a} f_{1}(t)\right]
$$

where, $\mathrm{X}_{1}$ is the positive sequence component

$X_{m}$ is the amplitude of the input signal

$\omega_{0}$ is the nominal frequency of the power system

$k_{x}$ is the magnitude step size

$k_{a}$ is the phase step size

$f_{l}(t)$ is a unit step function

Response time and delay time are defined in [5] subclause 5.3.3 and amended in [6]. Measurement delay time is evaluated in order to verify that time tagging of synchrophasors has been compensated for the group delay of the filtering system, such that the delay is near zero. An ideal step change is instantaneous by definition [5]; however, since the test signals may slew, the delay time is determined as the time when the stepped parameter achieves a value halfway between the starting and ending steady-state values.

It is worth noting that test signals are usually discrete time sampled at around $8 \mathrm{kHz}$; as such a minimum step time of $125 \mu \mathrm{s}$, equating to a phase angle of $2.25^{\circ}$ at $50 \mathrm{~Hz}$ or $2.7^{\circ}$ at $60 \mathrm{~Hz}$, is inevitable (this is taken into consideration in C37.118.1).

\section{Test Methodology}

In this paper the PMU under test is certified as C37.118-2005 compliant and has been established as such through in-house testing. We do not feel it is necessary to outline these tests as they are well established in existing literature [7], [11]. An overview of the method employed is presented in Fig. 2.

\subsection{Test waveform creation}

Three phase waveforms which represent the tests described in the subclauses of the IEEE standard have been created using both the Matlab and Python environment. The waveforms are modulated according to the parameters identified by each subclause. The nominal frequency can be set for $50 \mathrm{~Hz}, 60 \mathrm{~Hz}$ or any other arbitrary value, and the waveforms can be of any duration or sampling rate. The waveforms are exported as a 3-channel audio file in the commonly used Microsoft/IBM WAVE format (.wav), or Comma Separated Value (CSV), which can be interpreted by a variety of test equipment.

The equations in C37.118.1 describe dynamic theoretical phasors, with real and imaginary components. The algebraic phasor equations are turned into a discrete time series by incrementing the phasor by a discrete time step. The point on wave data is generated by recording the real component of the phasor at appropriate time intervals (usually 8 or $10 \mathrm{kHz}$ ); this point on wave data is saved as a CSV or WAVE file for loading into the relay tester.. The full phasor is also saved to a CSV file at appropriate time intervals $(50-60 \mathrm{~Hz})$ for comparison to the PMU output (derived from the point on wave replay).

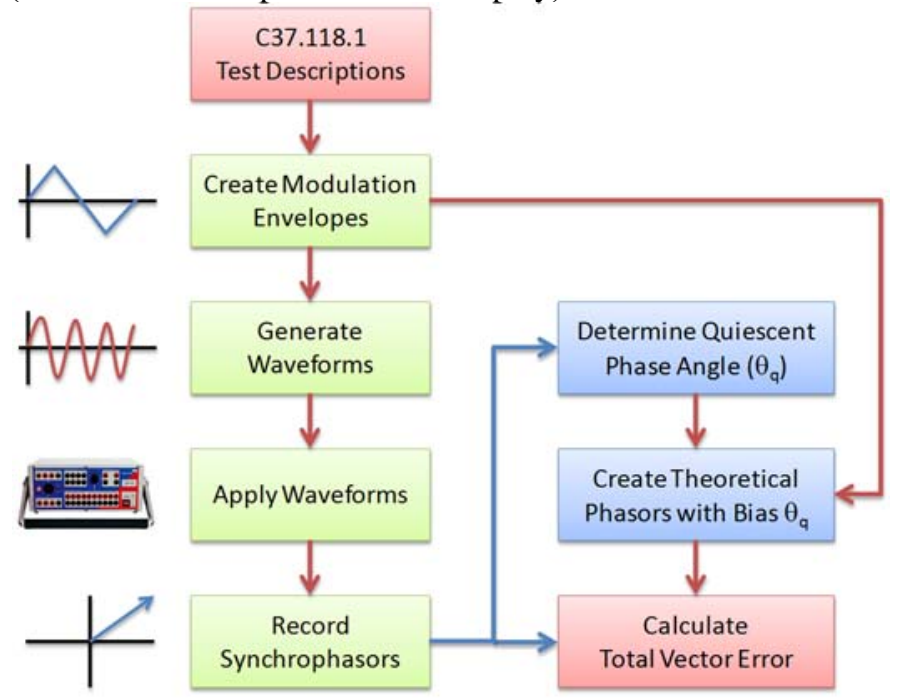

Fig. 2. Flow diagram describing the pre-compliance test methodology. A set of theoretical phasors (for comparison to the recorded phasors) are created alongside the point on wave files. The recorded phasors will not match the theoretical phasors due to temporal inaccuracy in replaying the waveform. The temporal error can be calculated from the recorded phase angle during the quiescent period (i.e. $18^{\circ}=1 \mathrm{~ms}$ at $50 \mathrm{~Hz}$ ). The temporal error is fed back into the code to generate a new set of theoretical phasors that account for the measured temporal error.

In this method, the WAVE/CSV file is created at the same time as the theoretical phasors described in [5]. The theoretical phasor is the magnitude and angle of the phasor that is creating the point on wave test file at the given moment in time. If perfect temporal accuracy was achieved in replaying the test files then the initial theoretical phasors could be used to test the accuracy of the PMU; but for commonly available equipment this is not the case. The frequency and rate of change of frequency, that drive the 
theoretical phasor, are also recorded and are necessary for C37.118.1 testing.

The test waveforms created contain a nominal lead in and lead out period, during which voltage magnitude should be nominal and phase angle equal to zero. The lead in period is employed to identify the delay in starting the test file and the lead out period is used to identify any temporal drift during the application of the test.

The Matlab and Python code used to create the test files are provided under an open source license via the OpenPMU project [12]. The software used to create the test files is under continued development and it is hoped that other researchers might benefit from this work, or contribute to further development.

\subsection{Applying Test Waveforms to PMU}

The resultant CSV or WAVE file was applied to a PMU via an 'Omicron CMC 156' protection relay test set. 'Test Universe' is the software used to control the Omicron 156 and it contains the package 'Trans Play' that can replay WAVE and CSV files. Software from manufacturers of other relay test equipment can provide similar functionality.

The test file was initiated with the leading edge of a 1-pulse per second input from an 'Omicron CMGPS' GPS time signal receiver applied to the Omicron CMC 156. This equipment suffers from a characteristic time delay of $1 \mathrm{~ms}$ on this channel and a sampling uncertainty of $0.1 \mathrm{~ms}$. In tests nominal wave forms thus applied had a phase angle of $18^{\circ} \pm 0.9^{\circ}$ at $50 \mathrm{~Hz}$. In theory no temporal synchronization is required as this is rectified through calibrating the theoretical phasors; however, a small, predictable temporal error is preferable to a random error as it aids data analysis.

In this test the output from the Omicron 156 was applied solely to the voltage inputs of the PMU under test. The purpose of this test is to identify intrinsic errors that arise in the estimation of phasors in a dynamic environment. By avoiding the use of CTs, and their potential inaccuracies, the ADC, time synchronization and the phasor estimation algorithm within the PMU are isolated.

While the temporal accuracy in initiating the waveform is low, the accuracy of the analogue outputs is high. The technical data on the Omicron CMC 156 claims accuracy in voltage and current output of $\pm 0.015 \%$. The contribution to TVE from the signal output error would then be in the region of $<0.02 \%$, this is $2 \%$ of the required minimum error of $1 \%$ TVE stipulated in [5].

\subsection{Recording PMU data}

The measurements made with the PMU are exported in the IEEE C37.118.2 data representation format [13]. For analysis, it was desirable to access the measurements in simple formats such as Comma Separated Values (CSV). We utilized the open source tool "PMU Connection Tester" [14].

\subsection{Calibrating the Theoretical Phasors}

The first step in the numerical analysis of the synchrophasor data involves the creation of a new set of theoretical phasors that are biased to correct for the time delay in starting the test file. The lead-in period of the signal is used to determine the quiescent phase angle, $\theta \mathrm{q}$, of the recorded synchrophasors. The quiescent phase angle can then be used to precisely identify the delay in starting the test file $\left(1^{\circ}=55.5 \mu\right.$ s at $50 \mathrm{~Hz}$, during the quiescent period).

The code that creates the WAVE, CSV and theoretical phasors can be biased in terms of its angle and magnitude. The phase angle and magnitude recorded during the quiescent period are thus used to create a new set of WAVE, CSV and theoretical phasors, only the theoretical phasors are of interest. In theory, the recorded phasors and the theoretical phasors should match exactly during the quiescent lead in period; this is exactly analogous to a PMU being calibrated to a nominal signal upon commissioning.

The method described isolates the TVE that arises due to dynamic operation; these errors are result from intrinsic PMU functions such as sampling time, dynamic accuracy and phasor estimation. The nominal behaviour at the end of the waveform allows any temporal drift to be identified, quantified and, if necessary, removed by slewing the theoretical phasors.

The testbench described is representative of standard relay test equipment. Equipment offering superior temporal accuracy is available in the marketplace, but can be prohibitively expensive and is not necessary for testing dynamics using the presented method.

\subsection{Numerical Analysis}

The accuracy of PMUs are tested, according to C37.118.1, with the TVE, frequency error, rate of change of frequency error, response time and delay time. Frequency error and rate of change of frequency error are simply calculated as the difference between the real and theoretical value. The TVE is calculated using Eqn. (1), when comparing the theoretical and measured phasor. The response and delay time are deduced from inspecting the step changes described below.

The numerical analysis can be carried out in any appropriate numerical environment (MS Excel, Matlab or Python). It is desirable to automate many of the processes, however it may be necessary to visually identify (or verify) the beginning of the test from the PMU output and identify the phase and magnitude error.

\subsection{Tests Applied to the PMU}

As outlined previously, three dynamic tests are specified in the C37.118.1 document and these are:

1) Measurement bandwidth

2) Ramp in frequency

3) Step change in phase / magnitude

(subclause 5.5.6) (subclause 5.5.7) (subclause 5.5.8)

Measurement bandwidth compliance waveforms are generated by modulating amplitude and phase angle. The modulation envelopes for $\mathrm{kx}=0.1$ and $\mathrm{ka}=0.1$ are presented in Fig. 3.

Frequency ramping is achieved in a similar way. Using a frequency ramp $\mathrm{Rf}=1.0 \mathrm{~Hz} / \mathrm{s}$, this yields a modulation envelope such as shown in Fig. 4.

Step change in phase and magnitude is achieved in much the same way. Fig. 5 shows the modulation envelopes to achieve step change in amplitude of $\mathrm{kx}=0.1$, and step change in phase $\mathrm{ka}=0.1 \mathrm{rad}$. Since the objective is to determine the PMU response / delay time to these events, these modulations would be applied independently. Using the technique of a modulation envelope eliminates concerns 
regarding discontinuities at the moment of the step change, since the fundamental tone otherwise continues to vary according to its original timebase.

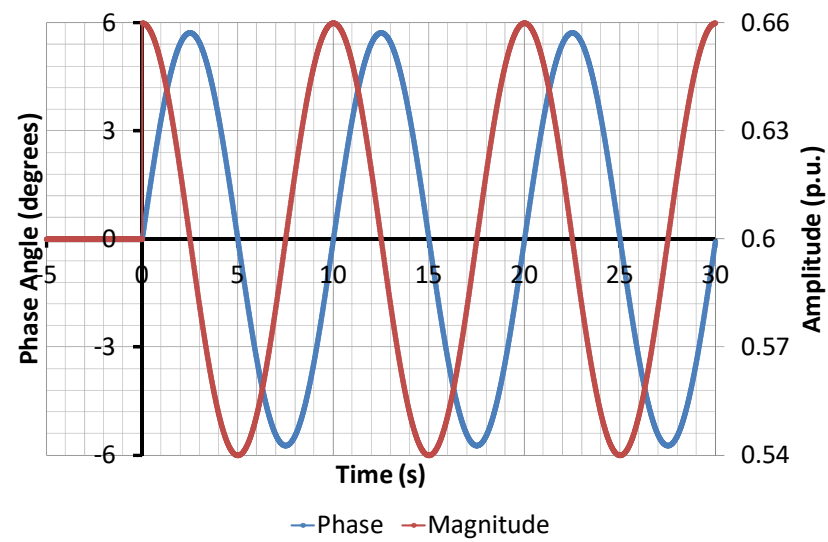

Fig. 3. Plot of the theoretical phasor amplitude and phase angle during the dynamic test 5.5.6 with modulation factors $k x=0.1$ or $k a=0.1 \mathrm{rad}$.

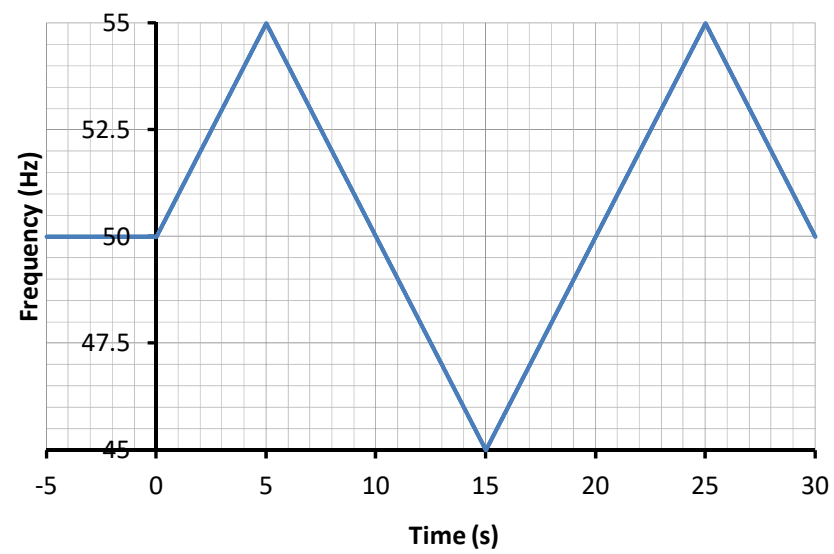

Fig. 4. Frequency envelope for theoretical phasor frequency during ramp test 5.5.7, $R f=1.0 \mathrm{~Hz} / \mathrm{s}$.

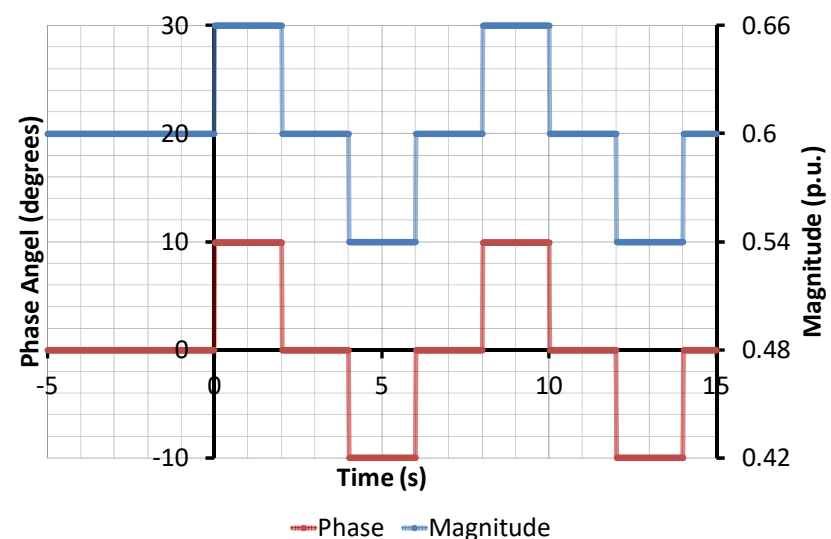

Fig. 5. Step change in phase and magnitude for the theoretically applied phasor in test 5.5.8, $\mathrm{kx}=0.1, \mathrm{ka}=10^{\circ}$ $(\pi / 18 \mathrm{rad})$.

\section{Results}

The compliance test was applied to a readily available commercial PMU that is in use with many European utilities. The results are presented in this paper to demonstrate the output of the pre-compliance testing method and are not to be taken as a critique of the actual PMU in question.

Both the theoretical synchrophasors and the measured synchrophasors were recorded in CSV format. Preprocessing steps include the removal of measurements pre- and post- test waveform playback, as well as discontinuities stipulated in [5]. The theoretical and recorded synchrophasors were then time aligned and compared. For each pair of synchrophasors, the frequency and rate of change of frequency $(d f / d t)$ error was easily calculated, likewise for phase and magnitude error; from which the TVE was determined, Eqn. (1).

As stated in the IEEE Std., a PMU can be classified as either $\mathrm{M}$ or P class; for the purposes of this investigation we decided to apply the most onerous tests specified in the C37.118.1 standard and judge the PMU from the results.

\subsection{Bandwidth Test - Sec. 5.5.6}

Test wave files were created with a modulation frequency between 0 and $5 \mathrm{~Hz}$. Between 0 and $2 \mathrm{~Hz}$ a test was conducted every $0.2 \mathrm{~Hz}$, as specified in the C37.118.1 standard. Between $2 \mathrm{~Hz}$ and $5 \mathrm{~Hz}$ a test was conducted every $0.5 \mathrm{~Hz}$, for ease of testing.

Fig. 6. Plot of total vector error against modulation frequency, as specified in the IEEE C37.118.1 standard Sec 5.5.6.

The C37.118.1 standard has specific requirements in relation to TVE, frequency error and rate of change of frequency error; shown in Fig. 6 is TVE against modulation frequency. It was assumed that TVE would increase linearly or exponentially with modulation frequency, however the measured TVE varies in a complex manner as modulation frequency increases. The noteworthy outcome is that the TVE never exceeds, or encroaches upon, the $3 \%$ limit set in [6], thus fulfilling all $\mathrm{P}$ and $\mathrm{M}$ class requirements.

The results for frequency error and ROCOF error (displayed in Table I) were more straightforward as the gradient of the error was always positive and generally linear; though step changes in gradient occurred. In this test the PMU passed the less onerous $\mathrm{M}$ class requirements for ROCOF, but fell far short of the stringent $P$ class requirements. In the frequency error test the PMU failed both the $\mathrm{P}$ and $\mathrm{M}$ class requirements, see Table I. 